\title{
Public Awareness of the Consanguinity and Genetic Disease Prevalence Connection in Saudi Arabia
}

\author{
Ohoud Ahmad Basuliman', Ghaydaa Faris Filimban², Ommamah Sami Alsabbah², \\ Khadijah Ahmad Alzhrani ${ }^{2}$, Miral Talal Fadhl' ${ }^{2}$, Ibrahim Abdulhafeez Mahbub ${ }^{2}$ and \\ Ahmad Hasan Mufti ${ }^{3^{*}}$ \\ 'Faculty of Medical Sciences, Umm Al-Qura University, Alabdiya - 24222, Makkah, Saudi Arabia; \\ basuliman.ohoud@gmail.com \\ 2Faculty of Medicine, Umm Al-Qura University, Alabdiya - 24222, Makkah, Saudi Arabia; \\ Ghaydaa.faris@gmail.com, ahla-mimo@hotmail.com,Khadijahahmad1995@gmail.com, \\ Miral.fadhl@gmail.com, Ibraheem_mahboob@hotmail.com \\ ${ }^{3}$ Faculty of Medicine, Umm Al-Qura University, Alabdiya - 24222, Makkah, Saudi Arabia; \\ ahmufti@uqu.edu.sa
}

\begin{abstract}
Consanguineous marriage is common in Saudi Arabia. It is responsible for high prevalence of genetic disorders in the Saudi population. Thus, the aim of this study is to assess the level of awareness around the genetic diseases resulting from consanguinity in Saudi Arabia. A randomised cross-sectional study was conducted among the Saudi society. A questionnaire was designed for demographics, the history of genetic diseases, awareness of genetic diseases and premarital screening. These were distributed through social media (Facebook, Twitter and WhatsApp), targeting different education levels and specialties, from different regions of the country. The data was analysed using the Statistical Package for Social Sciences (SPSS) and p-value calculated using a chi-square test. The majority of the participants were female, (83.4\%), versus (16.6\%) males. The participants were from different Saudi regions: the western (66.5\%), middle (11.5\%), southern (8.9\%), eastern $(7.8 \%)$ and northern (5.3\%) regions. $86 \%$ of the participants were undergraduates, $20.5 \%$ were educated to high-school level and $11.1 \%$ were postgraduate level. $28 \%$ were from a medical field while $72 \%$ were non-medical. The most common genetic diseases in the Saudi population are Down syndrome, sickle cell anemia, glucose-6-phosphate dehydrogenase (G6PD) deficiency and thalassemia. Participants with a medical education and those with higher educational levels demonstrate significantly higher levels of awareness about the connection between consanguinity and the resultant genetic diseases. Most of the participants believe that culture and tradition play the most important roles in the spread of the genetic diseases due to consanguinity, followed by a lack of awareness about their association. Public awareness of the association between consanguinity and the development of genetic diseases is relatively low. culture and tradition were reported to be most associated factor in the spread of consanguinity and thus genetic diseases.
\end{abstract}

Keywords: Awareness, Consanguinity, Genetic diseases, Saudi Arabia

${ }^{*}$ Author for correspondence 


\section{Introduction}

The word consanguineous comes from the two Latin words "con" meaning shared and "sanguis" meaning blood, thus describing a union and relationship between two individuals biologically related as second cousins or closer who share an ancestor or share blood ${ }^{1,2}$. Consanguineous marriage is a common phenomenon in the Middle East, especially in Muslim and tribal societies, but it has been associated with negative consequences for health, including reproductive health, fertility rate, birth defects, infant mortality rates or congenital anomalies that can lead to disability ${ }^{3}$.

The prevalence of consanguineous marriages varies between communities and populations, and it is depending on several factors, such as religion, culture and geographic distribution. The most prevalent type of consanguineous union is between first cousins. ${ }^{3}$ A study in Egypt shows that consanguineous marriages represent $31.4 \%$ for first cousins, $7.1 \%$ for one and a half cousins, $0.8 \%$ for double first cousins, $6 \%$ for second cousins and, $9.1 \%$ for more distant relations ${ }^{4}$. Consanguinity is recognized as the single cause of mortality resulting from genetic disorders ${ }^{5}$.

Genetic disorder is a pathological condition caused by changes to the DNA. It can be caused by a singlegene mutation, multiple-gene mutation, combined gene mutation, environmental factors or by a chromosomal abnormality ${ }^{6}$. Stillbirth, child death and recurrent abortion have a higher prevalence among consanguineous parents than among non-consanguineous parents ${ }^{4}$. This is because certain genetic disorders are inherited from the parents $^{7}$

There are several modes of inheritance: autosomal dominant, autosomal recessive, X-linked dominant, $\mathrm{X}$-linked recessive and single-gene disorders. Autosomal dominant disorder is transmitted to each generation; only one of the parents needs to be affected to transmit the disease to their offspring. An example of this type of inheritance is Huntington's Disease (HD) and Neurofibromatosis (NF). By contrast, for autosomal recessive diseases to be transmitted, a person must receive two copies of allele, that is from both parents; the parents can either have the disease or be carriers of it, such as is the case for sickle cell anemia and cystic fibrosis ${ }^{8}$. Research has shown that the most common inheritance pattern of consanguinity is the autosomal recessive disorder (78.8\%) and multifactorial disorders $(69.8 \%)^{4}$. This illustrates how consanguineous marriage increases the risk of spreading genetic diseases even if the disease phenotype is not expressed as such within their offspring since they can carry the trait.

A study was conducted in Saudi Arabia between 2004 and 2005, which found that there was a higher incidence of congenital heart disease as a consequence of consanguinity whereas a link with sickle cell anemia, G6PD deficiency and Down syndrome could not be established. ${ }^{9}$ A study conducted in Medina, Saudi Arabia, about the awareness of consanguineous marriage among 1,334 adults showed that consanguineous marriage is common in Medina, but there is a good awareness regarding the health hazards of consanguineous marriage, with good attitude towards the premarital screening ${ }^{10}$, Another study on the Saudi population focused on how to prevent genetic diseases resulting from the marriage of relatives. It found that the consanguinity rate is in excess of $50 \%$ with higher rate of inherited disease ${ }^{11}$.

Due to the wide spread of genetic diseases among the Saudi population, premarital screening has been introduced $^{12}$. They are a group of tests recommended for couples prior to get married to determine the risk of transmitting genetic diseases to their future offspring ${ }^{13}$. These types of test are available globally, but that each country tests for different diseases; in the case of Saudi Arabia, they test for sickle cell anemia and thalassemia.

There are a several benefits of these screenings: to limit the spread of some genetic blood diseases, and some infectious diseases including hepatitis B, C and AIDS/ HIV. This in turn reduces the financial burdens resulting from the treatment of these diseases. In addition to reduce the pressure over health institutions and blood banks and avoiding the social and psychological problems for families whose children suffer, making those seeking such as check-ups feel at ease, and also disseminating awareness with regard to the concept of the comprehensive, healthy marriage ${ }^{14}$.

A lack of knowledge in certain areas in Saudi Arabia has increased the spread of certain diseases, has led to suffering of the parents of the sick children and also increased the pressure on the state's health resources for the treatment. Thus, the objectives of this study are is to investigate the following amongst the participants: (1) to explore public awareness about genetic diseases resulting from consanguinity in Saudi Arabia, (2) to investigate the relationship between genetic disorders and consanguinity, (3) to provide an estimation as to the prevalence of 
consanguinity in the general population, (4) to compare awareness about consanguineous marriage between the different regions in Saudi Arabia, (5) to compare awareness about consanguineous marriage between people of different ages, genders, education levels, and those working in medical or non-medical field and (6) to assess knowledge about the prevention methods of genetic diseases, such as the use of premarital screening.

\section{Methods}

A randomised cross-sectional study was conducted among Saudi population. A questionnaire was designed by a study team to find out about the participants' demographics, genetic diseases, awareness about genetic diseases and premarital screening; it was then distributed electronically using social media such as Twitter and WhatsApp targeting participants from Saudi Arabia. This study used seven categories to filter the participants. A sample stratified by gender, age, 18 to 50 years old, marital status, nationality, different regions in Saudi Arabia, educational level, and whether they are from medical or non-medical field. Participants under 18 years old and non-Saudi were excluded from the study. 2,415 participants were selected from all those who answered the questionnaire. The confidentiality of all participants in this study was protected. Names of the participants was not be requested. No identification information was written or shown at all times, it was only be accessed by the authors. Informed consent was taken from all participants. IRB approval was obtained from King Abdullah Medical City (registration no. H-02-K-001) with IRB no. 18-441. The collected data was analyzed using the Statistical Package for Social Sciences (SPSS) with a chi-square test.

\section{Results}

The study included 2,650 participants; 235 participants were excluded as per the exclusion criteria. The total number of the participants that were female was 2,014 $(83.4 \%)$ while $401(16.6 \%)$ were male. $764(31.6 \%)$ of the participants were 18 to 25 years, 306 (12.7\%) 26 to 30 years, $276(11.4 \%) 31$ to 35 years, 319 (13.2\%) 36 to 40 years, $246(10.2 \%) 41$ to 45 years, 214 (8.9\%) 46 to 50 years and $290(12.0 \%)$ were above 50 years. Most of the participants were from the western region, 1,605 (66.5\%), and $278(11.5 \%)$ from the middle region, $216(8.9 \%)$ from the southern region, 188 (7.8\%) from the eastern region and $128(5.3 \%)$ from the northern region of Saudi Arabia. $1,529(63.3 \%)$ were married and $886(36.7 \%)$ were single. In terms of the educational level, most of the participants, 1,652 (68.4\%), had a university degree, followed by 496 (20.5\%) reaching high school and 267 (11.1\%) who were at postgraduate level; 673 (27.9\%) were from a medical field while $1,742(72.1 \%)$ were from a non-medical field.

Based on the results in this study, down syndrome, G6PD deficiency, thalassemia and Sickle Cell Anemia

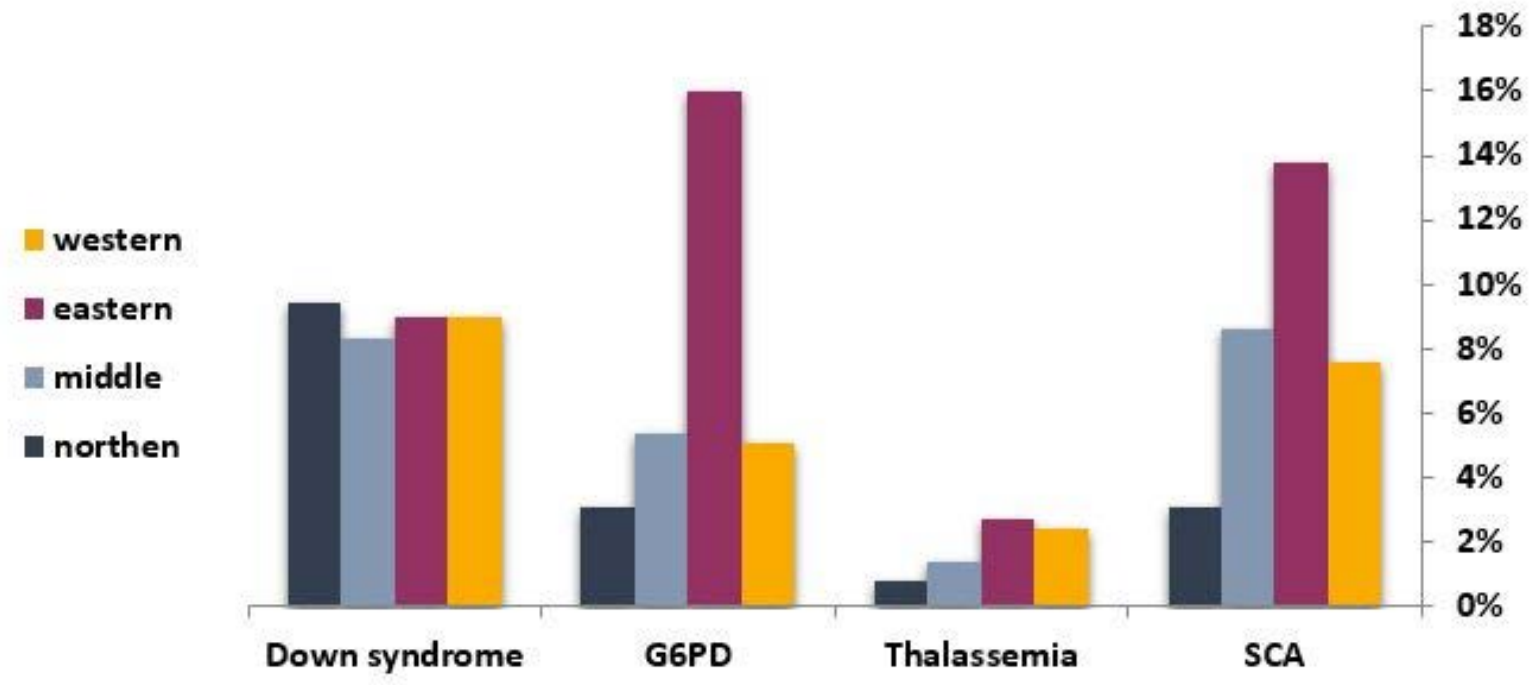

Figure 1. The most common genetic disease according to region in Saudi Arabia. 


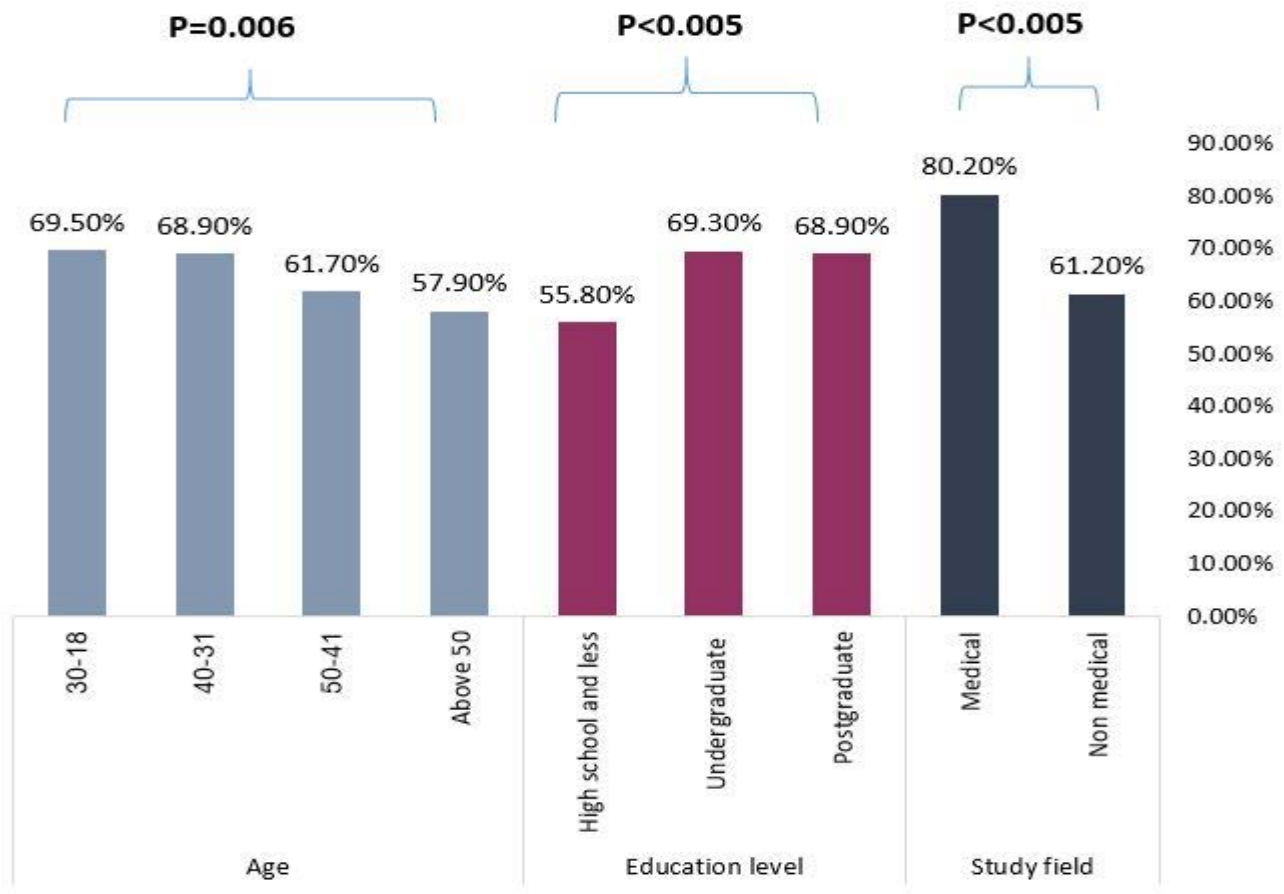

Figure 2. Participant awareness of genetic diseases resulting from consanguinity.

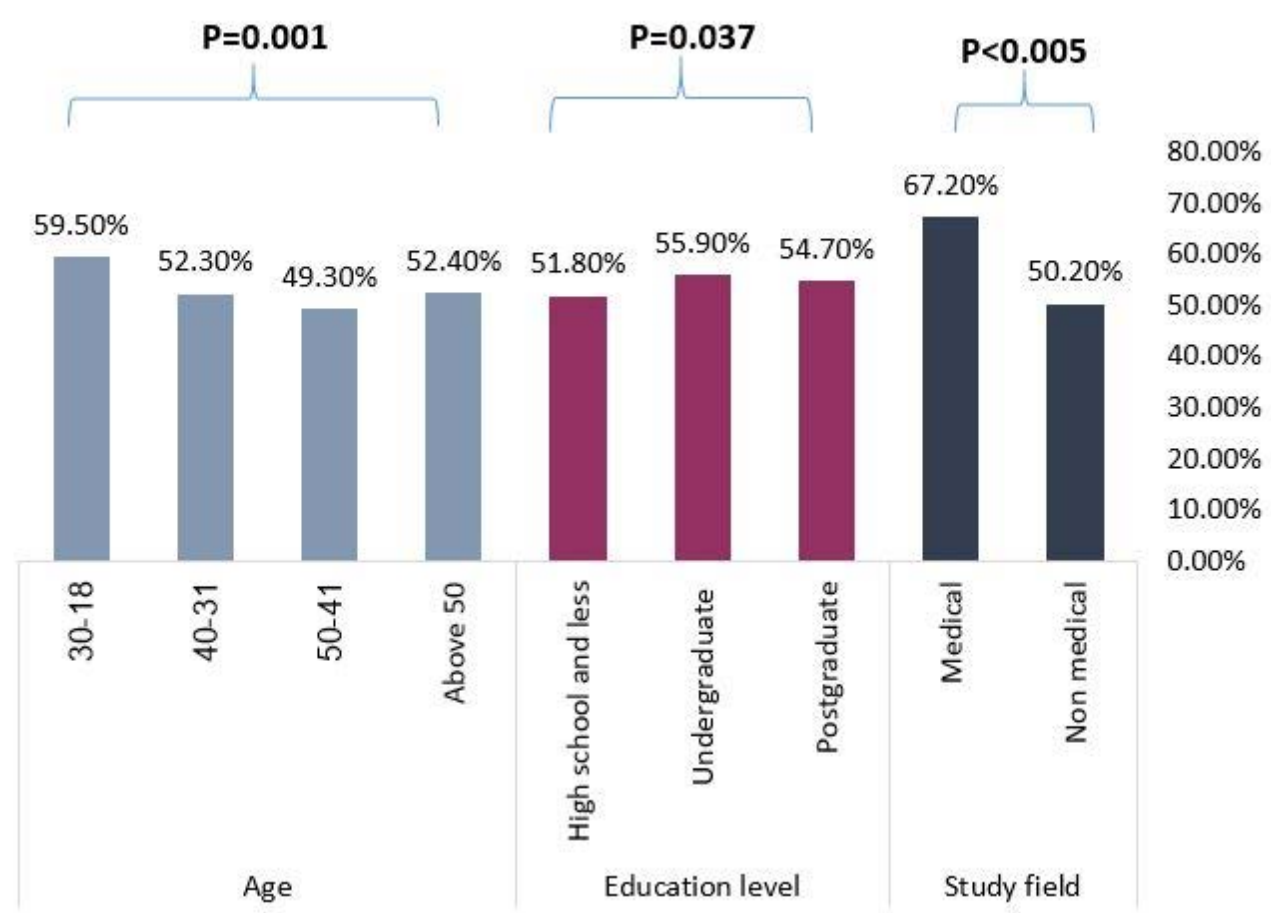

Figure 3. Participant opinions about consanguinity marriage as the main cause of the prevalence of genetic diseases. 


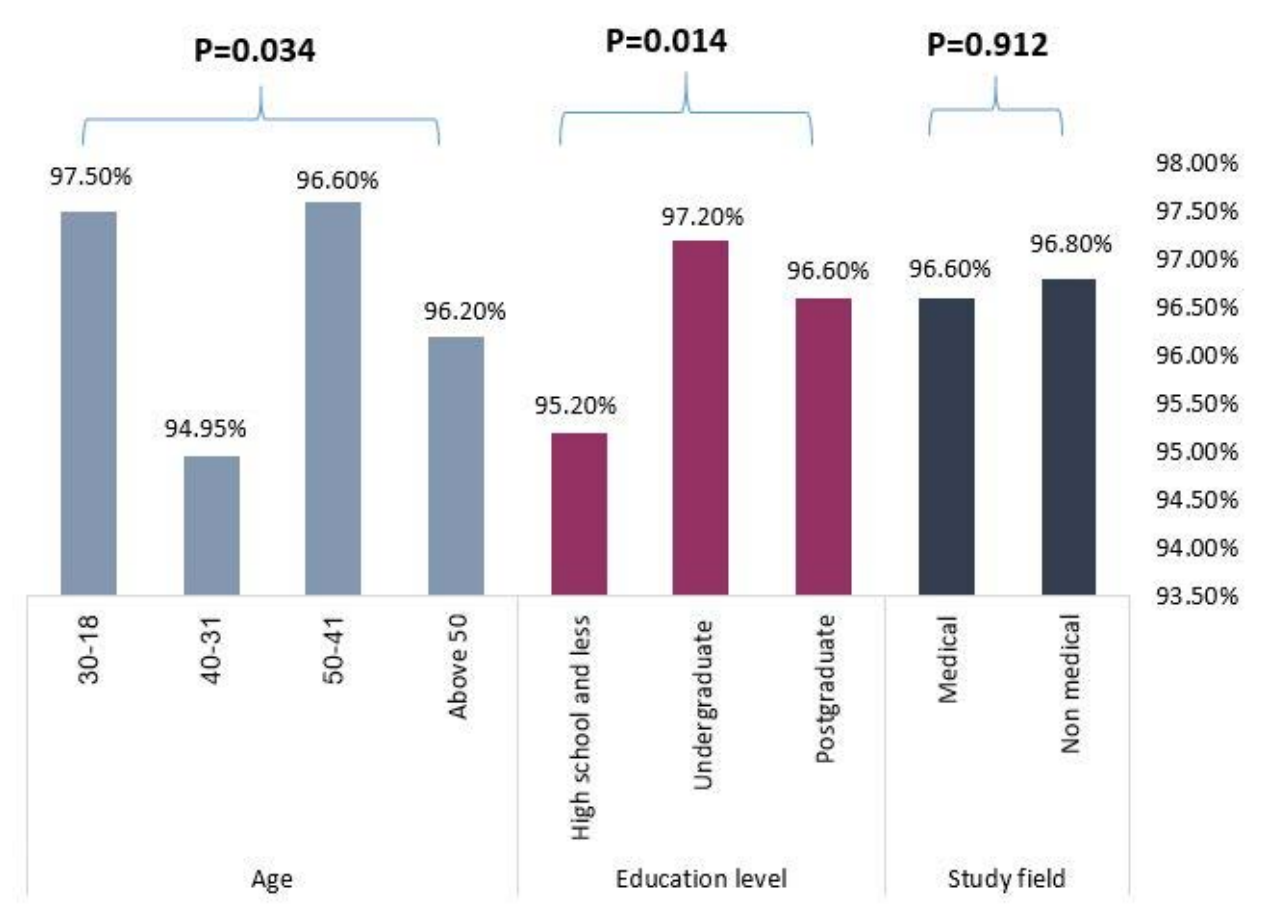

Figure 4. Participant awareness of premarital screening.

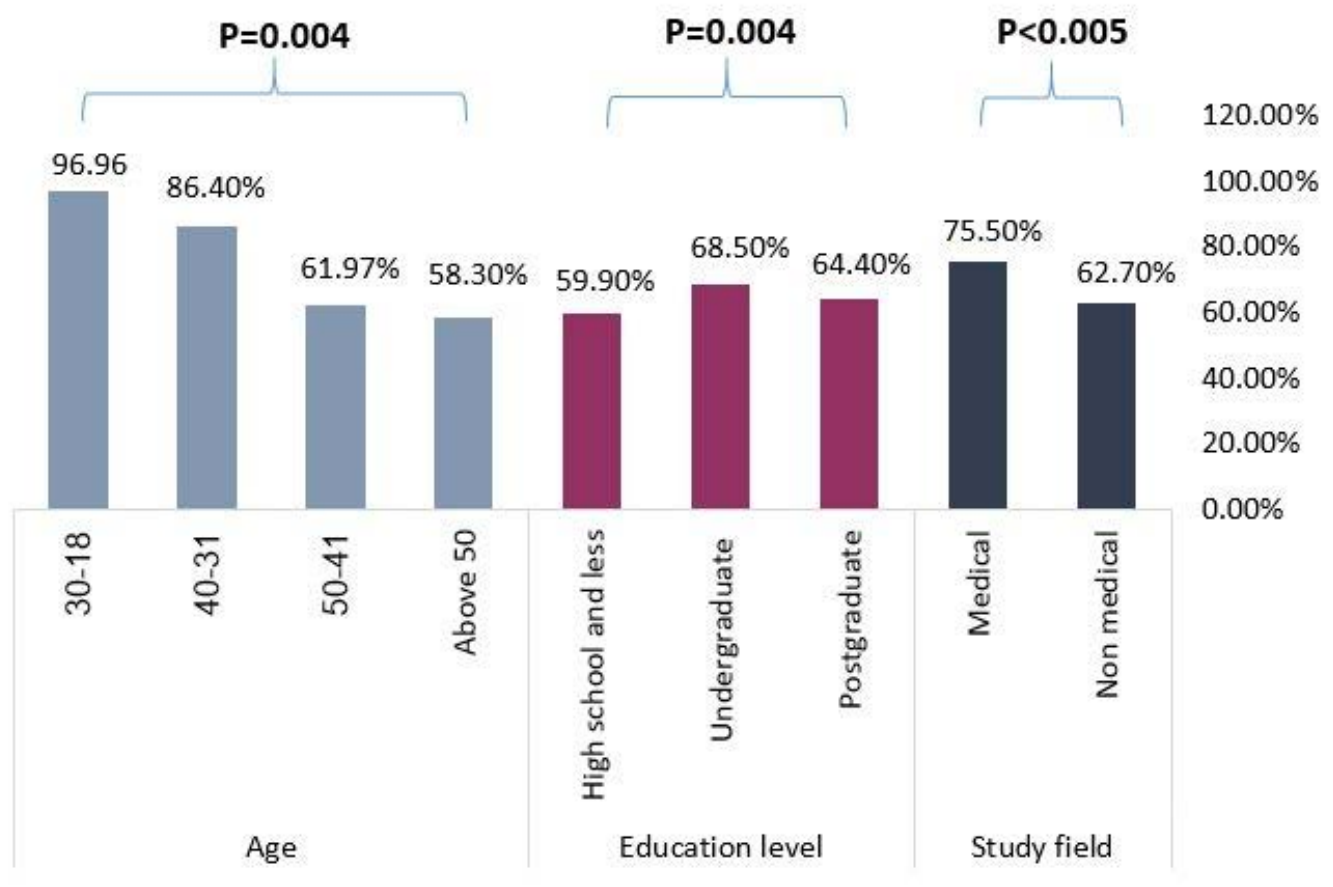

Figure 5. Participant awareness of how genetic diseases can be passed from parent to child. 


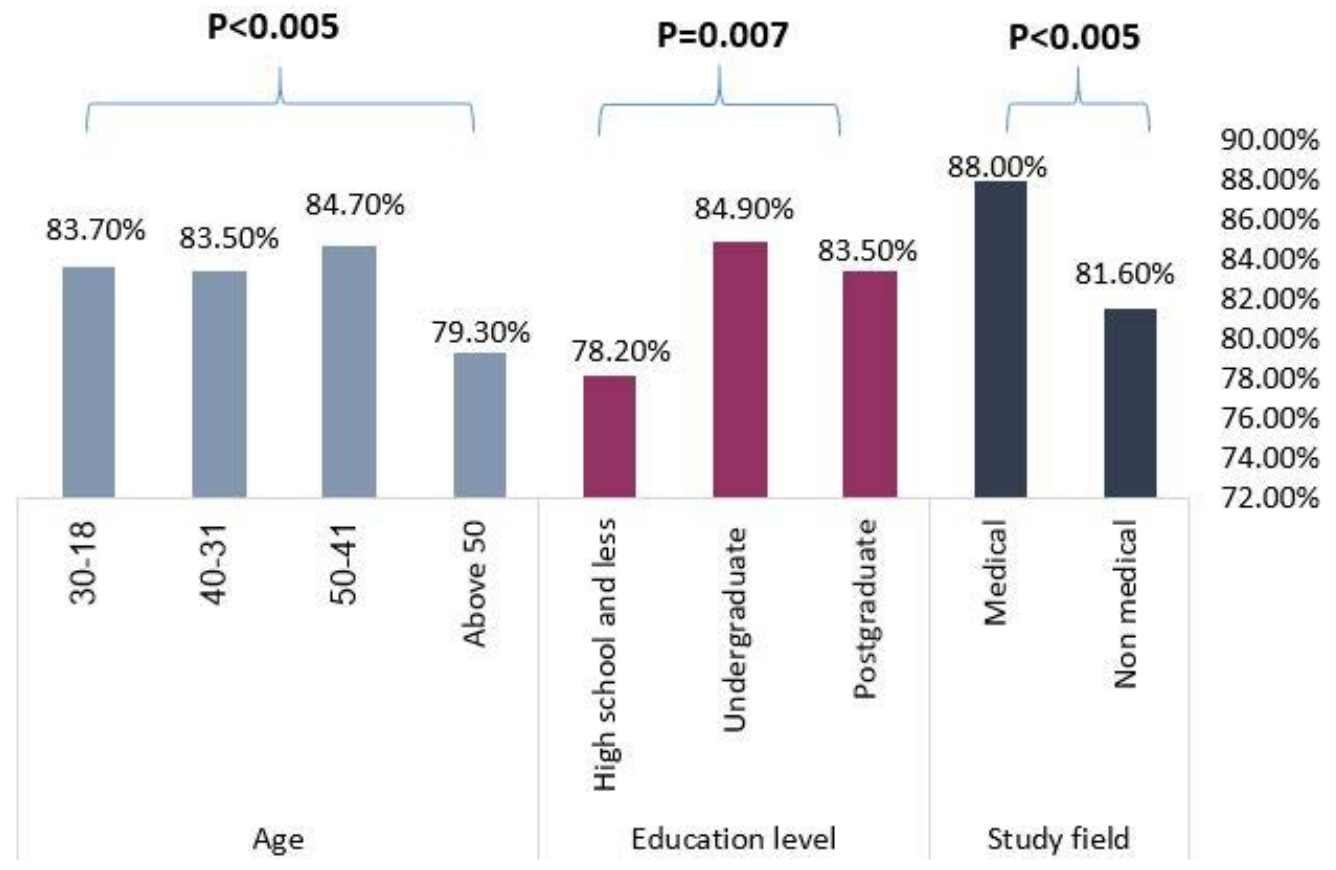

Figure 6. Participant awareness of genetic disease can affect the fetus.

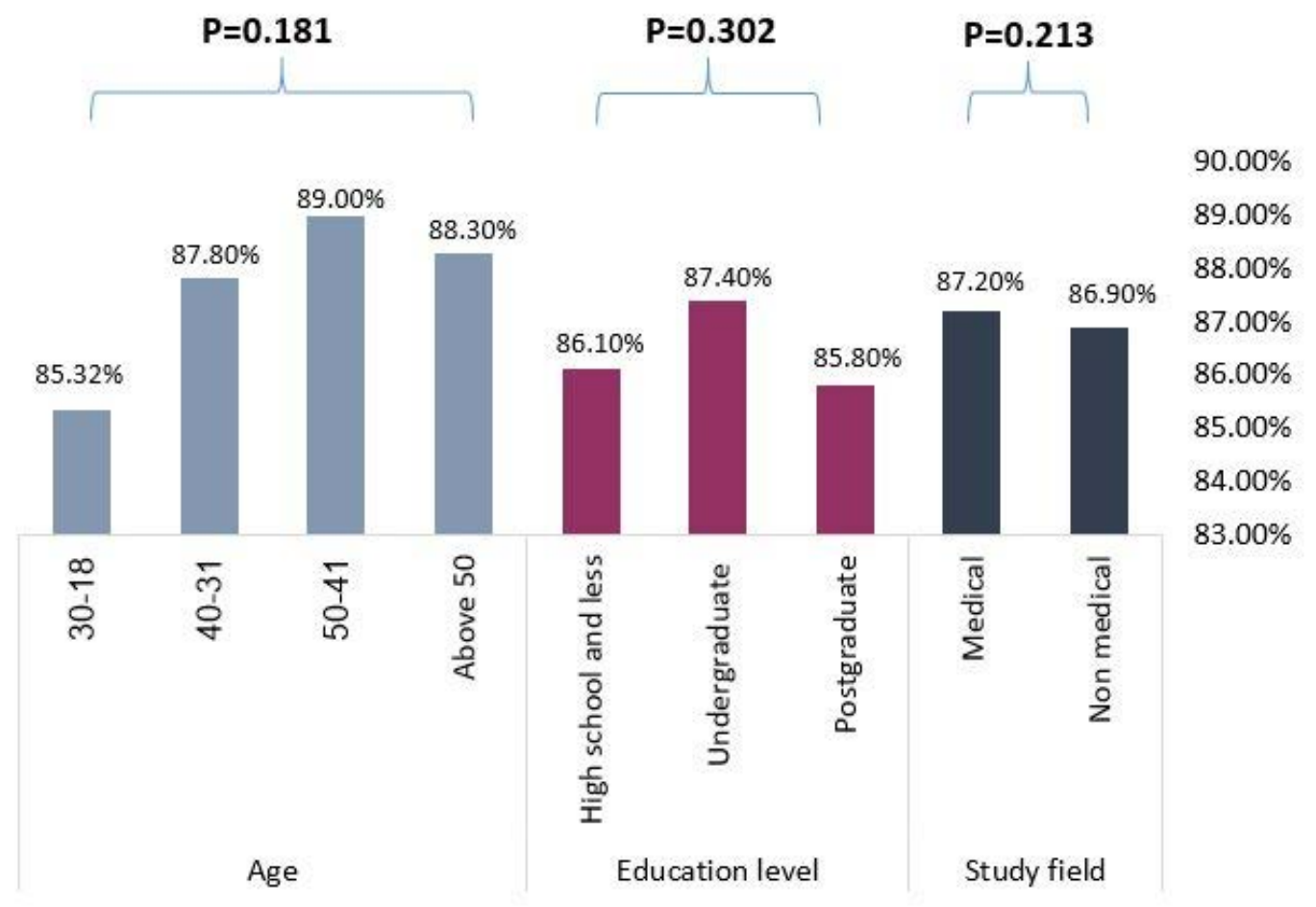

Figure 7. Participants who believe that medical consultation can avoid the occurrence of genetic diseases. 


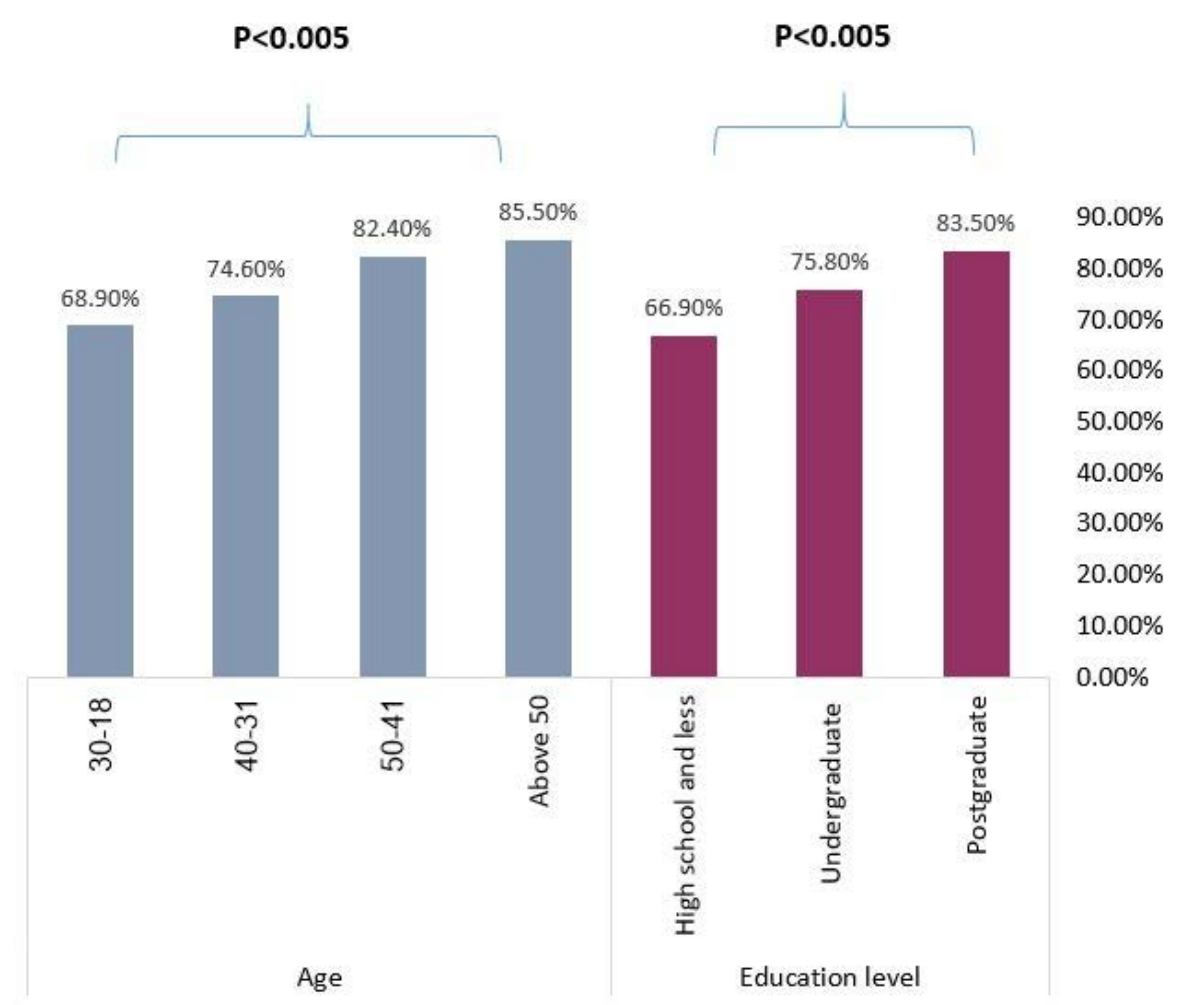

Figure 8. Participants who believe that genetic diseases are the cause for resource depletion.

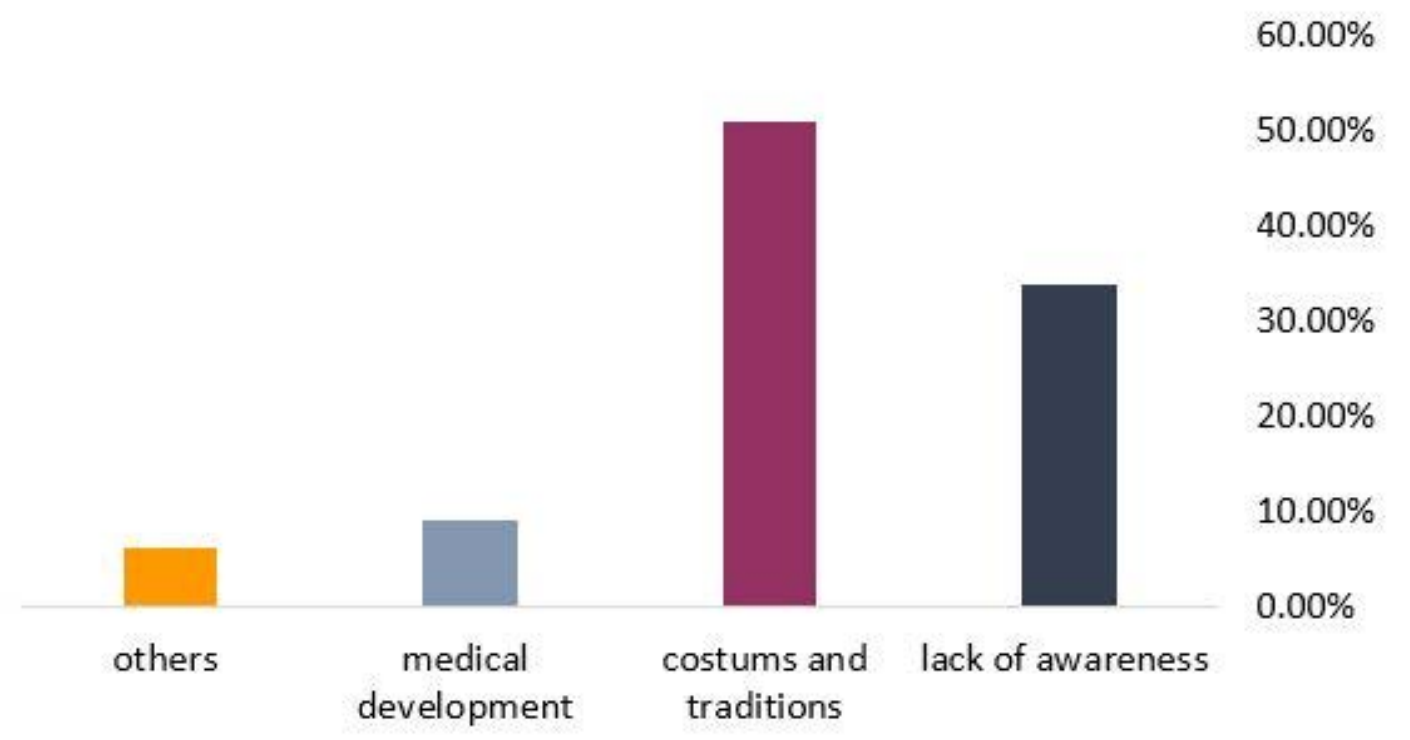

Figure 9. The cause of the high prevalence of consanguinity in Saudi Arabia according to the participants. 


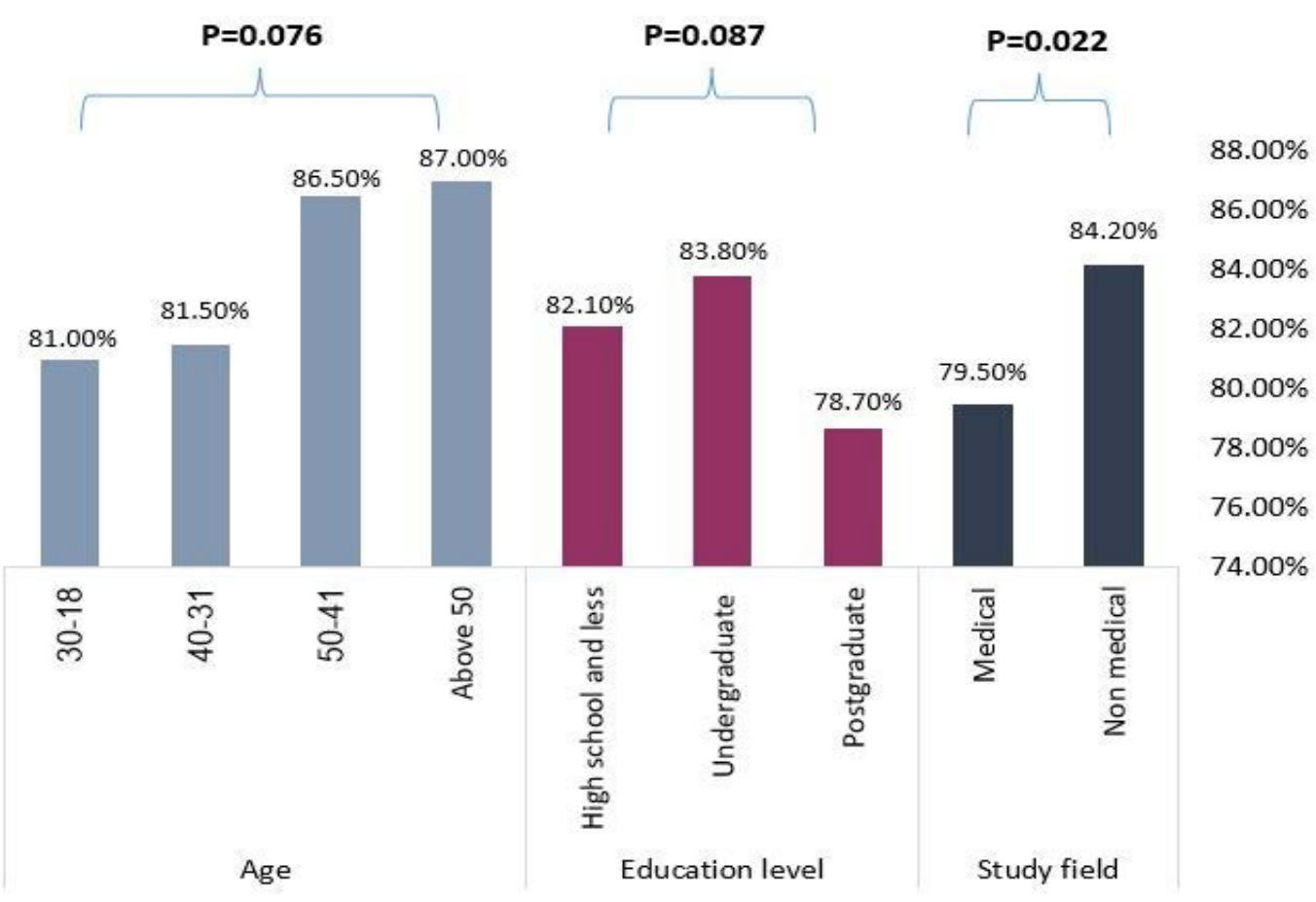

Figure 10. Participants who want to learn more about the pros and cons of consanguineous marriage.

(SCA) were the most common genetic diseases found in the participants of the study (see Figure 1). Down syndrome and thalassemia were found to be almost equally present in the different regions while G6PD was more common in the eastern region (16\%), which is associated with G6PD Mediterranean by very low intracellular enzyme activity ${ }^{15}$, SCA was found to be in the eastern (13.80\%) and southern regions (14.80\%); there is a large probability of a correlation between G6PD deficiency and SCA, which may lead to a more serious condition ${ }^{15}$. Moreover, it was found that the least common disease in the northern region was G6PD (3.10\%) and SCA (3.10\%) among participants (Figure 1).

Significantly higher percentage of participants in the 18-30 age range, and those with higher education levels or from a medical background claimed good awareness of the connection between consanguinity and genetic diseases (Figure 2). On the other hand, participants who were 18-30 years old, undergraduates and from a medical field believed that consanguinity is the main cause of the high prevalence of genetic diseases (Figure 3). Furthermore, they who were 18-30 years old, undergraduates believed in the importance of premarital screening to reduce the spread of genetic diseases (Figure 4). And who were 18-30 years old, were of aware the fact that genetic diseases can be passed from parents to children (Figure 5); they who were 18-30 years old also acknowledge that genetic disease can affect the fetus (Figure 6). Moreover, a high percentage of the participants between 41 and 45 years old, educated to undergraduate level, from a medical background, believe that medical consultation can provide different therapeutic approaches to reduce the occurrence of genetic diseases when parents are planning to have children (Figure 7).

Most participants above 50 years old, educated to a postgraduate level, from a medical background, agree that the spread of genetic diseases can lead to a depletion of government health resources (Figure 8); participants in the 31 to 40 age range, with an undergraduate or postgraduate qualification, but from a non-medical background support the idea of preventing marriage between those who have a genetic disease for fear of it being transferred to the fetus. Moreover, a majority of the participants over 50 years of age, with high school qualifications and from a non-medical background, did not know if there was a definitive cure for genetic diseases. Half of the participants believe that Saudi customs and traditions were the main cause of prevalence consanguinity marriage (50.9\%); 
followed by lack of awareness about the relationship between the consanguinity marriage and prevalence of genetic diseases (33.8\%) (Figure 9). Finally, the majority of participants expressed interest in being educated about the pros and cons of consanguineous marriage (Figure $10)$.

\section{Discussion}

Although the study population might not be representative of the Saudi population, it showed widespread incidence of consanguineous marriage in Saudi Arabia, and the eastern and southern regions have the highest prevalence of genetic diseases amongst the participants of this study, which is in line with reports from the Ministry of Health in KSA. In other study which conducted in Saudi Arabia shows that the parents of children affected with genetic diseases had higher proportion of consanguinity in the, reaching what might be considered a borderline statistical significance. ${ }^{16}$ The majority of the participants were from younger age ranges ( 35 years old or less), which is expected since the questionnaire was distributed online, and this group of individuals use social media the most. Education levels, awareness, culture and tradition are all compounding factors in the spread of genetic diseases according to the participants' opinion. Just over half of the participants, $50.90 \%$, believed that customs and traditions are the major cause of the prevalence of consanguinity marriage. The younger participants between 18-30 years old were more aware that consanguinity can be associated with inherited genetic disease. Moreover, most of the older participants above 50 years old did not know whether genetic diseases are treatable or not. It can be seen that awareness increases with younger participants, and this is because education has become better and more developed in last few years in addition to all the effort the government is doing to reduce the spread of genetic diseases. The result has shown that awareness increases with high levels of education because better education translates to better knowledge. The participants who were 18-30 years old believe that consanguinity is the main cause of the high prevalence of genetic diseases were also aware of the importance of premarital screening to reduce the spread of genetic diseases. The other study reported that there was a good knowledge about the nature of the premarital tests among participants and about the targeted disorders. Moreover, higher than $80 \%$ of participants believing that it should include both sexually and genetically transmitted diseases. And more than $60 \%$ of all the participants were in favor of preventing at-risk marriages. ${ }^{17}$ Moreover, these participants who were 18-30 years old aware that genetic diseases can be passed from parents to children which support our previous claim. On the other hand, it could be said that the older participants have better knowledge of the impact of genetic diseases on the economy as a result, they support preventing marriage if the couple is found to have a genetic disease since it could be transferred to their children. In addition, they believe that genetic diseases are the cause for resources depletion.

\section{Conclusion}

Culture and tradition were chosen as the most important factors in spreading of consanguineous marriage and thus genetic diseases by the participants, a lack of awareness of the risk of such type of marriage seems to be the cause of retaining such tradition. However, Current data show raising awareness and understanding of the risk of consanguineous marriage and its impact on the spread of genetic diseases since younger participants showed better awareness. Yet, better public awareness would contribute to further reduction in children with genetic diseases either through reducing the number of consanguineous marriages or through acknowledging the importance and following the results of premarital testing.

\section{References}

1. Kanaan ZM, Mahfouz R, Tamim H. The prevalence of consanguineous marriages in an underserved area in Lebanon and its association with congenital anomalies. Genetic testing. 2008; 12(3): 367-72. https://doi.org/10.1089/ gte. 2007.0093

2. Hamamy H. Consanguineous marriages : Preconception consultation in primary health care settings. J Community Genet. 2012; 3(3): 185-92. https://doi.org/10.1007/s12687011-0072-y

3. Hamamy H. Consanguineous marriages: preconception consultation in primary health care settings. J. Community Genet. 2012; 3(3):185. https://doi.org/10.1007/s12687-0110072-y

4. Shawky RM, Elsayed SM, Zaki ME, El-Din SMN, Kamal FM. Consanguinity and its relevance to clinical genetics. Egypt. J. Med. Hum. Genet. 2013; 14(2). https://doi. org/10.1016/j.ejmhg.2013.01.002 
5. Akram D, Arif F, Fayyaz J. How frequent are consanguineous marriages? JDUHS. 2008; 2(2): 76-9.

6. FAQ About Genetic Disorders 2015 [updated November 10, 2015. Available from: https://www.genome.gov/19016930/ faq-about-genetic-disorders/.

7. Boycott KM, Vanstone MR, Bulman DE, MacKenzie AE. Rare-disease genetics in the era of next-generation sequencing: discovery to translation. Nature Reviews Genetics. 2013; 14(10): 681-91. https://doi.org/10.1038/ nrg3555

8. Genetic NY-M-ACf, Services NS. Understanding genetics: a New York, mid-Atlantic guide for patients and health professionals: Lulu. com; 2009.

9. El Mouzan MI, Al Salloum AA, Al Herbish AS, Qurachi $\mathrm{MM}, \mathrm{Al}$ Omar AA. Consanguinity and major genetic disorders in Saudi children: a community-based cross-sectional study. Annals of Saudi medicine. 2008; 28(3): 169-73. https://doi.org/10.5144/0256-4947.2008.169

10. Alharbi KM, Alharbi TM, Alhejaili ME, Alsharif AA, Alshenaifi RF, Zahid BN, et al. Awareness Regarding Health Hazards of Consanguineous Marriage among Saudi Adults in Medina, 2016. Age (years). 20(162): 12.1.

11. Meyer BF. Strategies for the prevention of hereditary diseases in a highly consanguineous population. Annals of Human Biology. 2005; 32(2): 174-9. https://doi. org/10.1080/03014460500075217
12. Memish ZA, Saeedi MY. Six-year outcome of the national premarital screening and genetic counseling program for sickle cell disease and $\beta$-thalassemia in Saudi Arabia. Annals of Saudi medicine. 2011; 31(3): 229. https://doi. org/10.4103/0256-4947.81527

13. Memish ZA, Saeedi MY. Six-year outcome of the national premarital screening and genetic counseling program for sickle cell disease and beta-thalassemia in Saudi Arabia. Ann Saudi Med. 2011; 31(3): 229-35. https://doi. org/10.4103/0256-4947.81527

14. Premarital Screening. https://www.moh.gov.sa/en/Health Awareness/EducationalContent/PublicHealth/Pages/ PremaritalScreening.aspx Minstery of health, Saudi Arabia

15. Al-Ali A. Common G6PD variant from Saudi population and its prevalence. Annals of Saudi medicine. 1996; 16(6): 654-6. https://doi.org/10.5144/0256-4947.1996.654

16. Mouzan MIE, Salloum AAA, Herbish ASA, Qurachi MM, Omar AAA. Consanguinity and major genetic disorders in Saudi children: a community-based cross-sectional study. Annals of Saudi Medicine. 2008; 28(3): 169-73. https://doi. org/10.5144/0256-4947.2008.169

17. Binshihon SM, Alsulami MO, Alogaibi WM, Mohammedsaleh AH, Mandourah HN, Albaity BS, et al. Knowledge and attitude toward hemoglobinopathies premarital screening program among unmarried population in western Saudi Arabia. Saudi medical journal. 2018; 39(12): 1226-31. https://doi.org/10.15537/smj.2018.12.23136 